\title{
Application of the SBA Method for Solving the Partial Differential Equation
}

\author{
Gires Dimitri NKAYA ${ }^{1}$, Francis BASSONO ${ }^{2}$, Rasmané YARO ${ }^{2}$, Joseph BONAZEBI YINDOULA $^{1} \&$ Gabriel $^{2}$ \\ BISSANGA ${ }^{1}$ \\ ${ }^{1}$ University Marien NGOUABI, Technology and Faculty of Science Brazzaville, Congo \\ ${ }^{2}$ University of Ouaga-1 Professeur Joseph KI ZERBO, Burkina Faso
}

Correspondence: Joseph BONAZEBI YINDOULA, University Marien NGOUABI,Technology and Faculty of Science, Brazzaville, Congo. E-mail: bonayindoula@yahoo.fr

Received: July 5, 2018 Accepted: November 28, 2018 Online Published: November 28, 2018

doi:10.5539/jmr.v10n6p98 URL: https://doi.org/10.5539/jmr.v10n6p98

\begin{abstract}
In this paper, the SBA method is used to construct the solution of the nonlinear partial differential equations.
\end{abstract}

Keywords: SBA method, wave-like equation, partial differential equation.

\section{Introduction}

The nonlinear problem play a significant role in many diverse areas of science and technology. Many problem are gouverned by partial differential equations, or by systems of partial differential equations. It is difficult to find their exact solutions. In this paper, we use the Some Blaise Abbo (SBA) method (Abbaoui. K and Cherruault. Y (1999); Abbaout. K and Cherruault. Y .(1995); Abbo Bakari (2007); Bonazebi Yindoula J., Pare Youssouf, Bissanga Gabriel, Bassono Francis and Some Blaise, (2014); Pare Youssouf, (2010); Pare Youssouf, Yaro Rasmane, Elysee Gouba and Blaise Some,(2012))to find the exact solution of some wave-like equations with variable coefficients ( Ghoreishi, M., Ismail, A. I. B. and Ali, N. H. M. (2010); V.G.Gupta, Sumit Gupta (2013)) and a system of partial differential equations (Ghoreishi, M., Ismail, A. I. B. and Ali, N. H. M. (2010); Wazwaz, A.M.,(2002)). These equation have been studied in (Ghoreishi, M., Ismail, A. I. B. and Ali, N. H. M. (2010)) is (V.G.Gupta, Sumit Gupta (2013)), one used the homotopy perturbation to get the approached solution for the wave-like equations and the exact solution for the system of partial differntial equations.

\section{About the SBA Method}

Let's consider the following fonctional equation

$$
A u=f
$$

Where $A: H \longrightarrow H$, is a linear or nonlinear operator and $H$ is a Hilbert space.

Let's suppose that we can decompose the nonlinear in following form :

$$
A=L-R-N
$$

Where $L+R$ is linear , $N$ nonlinear, $L$ is an inversible in the Adomian sense.

Equation (1) therefore becomes :

$$
L u-R u-N u=f \Longleftrightarrow u=\theta+L^{-1}(f)+L^{-1}(R u)+L^{-1}(N u)
$$

Where $\theta$ is such that $L \theta=0$. Equation (3) is the Adomian canonical form (ABBAOUI. K and CHERRUAULT. Y .(1995)). Using the successive approximations (ABBO BAKARI (2007)), we get

$$
u^{k}=\theta^{k}+L^{-1}\left(f^{k}\right)+L^{-1}\left(R u^{k}\right)+L^{-1}\left(N u^{k-1}\right) ; \quad k \geqslant 1
$$

This vields the following Adomian algorithm:

$$
\left\{\begin{array}{l}
u_{0}^{k}=\theta^{k}+L^{-1}\left(f^{k}\right)+L^{-1}\left(N\left(u^{k-1}\right)\right) ; \quad k \geq 1 \\
u_{n}^{k}=L^{-1}\left(R u_{n-1}^{k}\right) ; \quad n \geq 1
\end{array}\right.
$$


If the series

$$
\sum_{n=0}^{+\infty} u_{n}^{k}(x, t)
$$

converges, then

$$
u^{k}=\sum_{n=0}^{+\infty} u_{n}^{k}(x, t)
$$

, therefore

$$
u=\lim _{k \rightarrow+\infty} u_{n}^{k}
$$

is the solution of the equation (1).

\section{Numerical Applications}

\section{Example 1}

Consider the following nonlinear wave-like equation with variable coefficients(Ghoreishi, M., Ismail, A. I. B. and Ali, N. H. M. (2010))

$$
\left\{\begin{aligned}
\frac{\partial^{2} u(x, t)}{\partial t^{2}} & =N(u(x, t))-u(x, t), \quad 0<x<1, \quad t>0 \\
u(x, 0) & =0 \\
u_{t}(x, 0) & =x^{2}
\end{aligned}\right.
$$

With

$$
N(u(x, t))=x^{2} \frac{\partial}{\partial x}\left(u_{x} u_{x x}\right)-x^{2}\left(u_{x x}^{2}\right)
$$

From (8) we obtain the following canonical Adomian form :

$$
u(x, t)=x^{2} t+\int_{0}^{t}\left(\int_{0}^{s} N(u(x, z)) d z\right) d s-\int_{0}^{t}\left(\int_{0}^{s} u(x, z) d z\right) d s
$$

Let us applay to (10) the method of the successive approximations, one obtains

$$
u(x, t)=x^{2} t+\tilde{N}\left(u^{k-1}\right)-\int_{0}^{t}\left(\int_{0}^{s} u(x, z) d z\right) d s
$$

Where

$$
\tilde{N}\left(u^{k-1}\right)=\int_{0}^{t}\left(\int_{0}^{s} N\left(u^{k-1}(x, z)\right) d z\right) d s
$$

From (11), we have the following algorithm of Adomian:

$$
\left\{\begin{array}{l}
u_{0}^{k}=x^{2} t+\tilde{N}\left(u^{k-1}\right) ; \quad k \geq 1 \\
u_{n}^{k}=-\int_{0}^{t}\left(\int_{0}^{s} u_{n-1}^{k}(x, z) d z\right) d s ; \quad n \geq 1
\end{array}\right.
$$

First step

For $k=1$, we take $u^{0}(x, t)=0$ and we obtain $\tilde{N}\left(u^{0}\right)=0$. 
From(13), we have

$$
\left\{\begin{aligned}
u_{0}^{1} & =x^{2} t \\
u_{1}^{1} & =(-1)^{1} \frac{t^{3}}{3 !} x^{2} \\
u_{2}^{1} & =(-1)^{2} \frac{t^{5}}{5 !} x^{2} \\
u_{3}^{1} & =(-1)^{3} \frac{t^{7}}{7 !} x^{2} \\
\cdot & \\
\cdot & \\
u_{n}^{1} & =(-1)^{n} \frac{t^{2 n+1}}{(2 n+1) !} x^{2}
\end{aligned}\right.
$$

Thus the exact solution to the first step is

$$
u^{1}(x, t)=\sum_{n=0}^{\infty} u_{n}^{1}=x^{2}\left(\sum_{n=0}^{\infty}(-1)^{n} \frac{t^{2 n+1}}{(2 n+1) !}\right)=x^{2} \sin t
$$

Second step

For $k=2$, we have

$$
\left\{\begin{aligned}
\tilde{N}\left(u^{1}(x, t)\right) & =\int_{0}^{t}\left(\int_{0}^{s}\left(x^{2} \frac{\partial}{\partial x}\left(u_{x}^{1} u_{x x}^{1}\right)-x^{2}\left(u_{x x}^{1}\right)^{2}\right) d z\right) d s \\
& =\int_{0}^{t}\left(\int_{0}^{s}\left(4 x^{2} \sin ^{2} z-4 x^{2} \sin ^{2} z\right) d z\right) d s=0
\end{aligned}\right.
$$

From (13), we obtain

$$
\left\{\begin{aligned}
u_{0}^{2} & =x^{2} t \\
u_{1}^{2} & =(-1)^{1} \frac{t^{3}}{3 !} x^{2} \\
u_{2}^{2} & =(-1)^{2} \frac{t^{5}}{5 !} x^{2} \\
u_{3}^{2} & =(-1)^{3} \frac{t^{7}}{7 !} x^{2} \\
\cdot & \cdot \\
\cdot & =(-1)^{n} \frac{t^{2 n+1}}{(2 n+1) !} x^{2}
\end{aligned}\right.
$$

and

$$
u^{2}(x, t)=\sum_{n=0}^{\infty} u_{n}^{2}=x^{2}\left(\sum_{n=0}^{\infty}(-1)^{n} \frac{t^{2 n+1}}{(2 n+1) !}\right)=x^{2} \sin t
$$

From wich, we obtain

$$
u^{k}(x, t)=x^{2} \sin t
$$


From wich, we obtain

$$
u^{k}(x, t)=\lim _{k \rightarrow+\infty} u_{n}^{k}=x^{2} \sin t
$$

Example 2 Consider the following two dimensional nonlinear wave-like equation with variable coefficients ( Ghoreishi, M., Ismail, A. I. B. and Ali, N. H. M. (2010))

$$
\left\{\begin{aligned}
\frac{\partial^{2} u(x, y, t)}{\partial t^{2}} & =N(u(x, y, t))-u(x, y, t), \quad 0<x<1, \quad t>0 \\
u(x, y, 0) & =e^{x y} \\
u_{t}(x, y, 0) & =e^{x y}
\end{aligned}\right.
$$

With

$$
N(u(x, y, t))=\frac{\partial^{2}}{\partial x \partial y}\left(u_{x x} u_{y y}\right)-\frac{\partial^{2}}{\partial x \partial y}\left(x y u_{x} u_{y}\right)
$$

From(21) we obtain the following canonical Adomian form

$$
u(x, y, t)=e^{x y}+t e^{x y}+\int_{0}^{t}\left(\int_{0}^{s} N(u(x, y, z)) d z\right) d s-\int_{0}^{t}\left(\int_{0}^{s} u(x, y, z) d z\right) d s
$$

From(23), the successive approximations give us

$$
u^{k}(x, y, t)=e^{x y}+t e^{x y}+\tilde{N}\left(u^{k-1}(x, y, z)\right)-\int_{0}^{t}\left(\int_{0}^{s} u^{k}(x, y, z) d z\right) d s
$$

Where

$$
\tilde{N}\left(u^{k-1}(x, y, z)\right)=\int_{0}^{t}\left(\int_{0}^{s} N\left(u^{k-1}(x, y, z)\right) d z\right) d s
$$

From (24) we have the following algorithm of Adomian:

$$
\left\{\begin{aligned}
u_{0}^{k} & =e^{x y}+t e^{x y}+\tilde{N}\left(u^{k-1}(x, y, z)\right), \quad, k \geqslant 1 \\
u_{n+1}^{k} & =-\int_{0}^{t}\left(\int_{0}^{s} u_{n}^{k}(x, y, z) d z\right) d s \quad, n \geqslant 0
\end{aligned}\right.
$$

Let us apply to (26), the principle of picardy, we remark that $u^{0}(x, y, t)=0$ is a root of the $\tilde{N}\left(u^{0}(x, y, z)\right)=0$ And for $k=1$, we obtain :

$$
\left\{\begin{array}{l}
u_{0}^{1}=e^{x y}+t e^{x y} \\
u_{1}^{1}=e^{x y}\left(-\frac{t^{2}}{2 !}-\frac{t^{3}}{3 !}\right) \\
u_{2}^{1}=e^{x y}\left(\frac{t^{4}}{4 !}+\frac{t^{5}}{5 !}\right) \\
u_{3}^{1}=e^{x y}\left(-\frac{t^{6}}{6 !}-\frac{t^{7}}{7 !}\right) \\
\cdot \quad \\
\cdot \quad e^{x y}\left[(-1)^{n} \frac{t^{2 n}}{(2 n) !}+(-1)^{n} \frac{t^{2 n+1}}{(2 n+1) !}\right]
\end{array}\right.
$$

let's put

$$
\varphi_{m}(x, y, t)=\sum_{n=0}^{m-1} u_{n}^{1}(x, y, t)=e^{x y}\left(\left[(-1)^{n} \frac{t^{2 n}}{(2 n) !}+(-1)^{n} \frac{t^{2 n+1}}{(2 n+1) !}\right]\right)
$$


From (28), we obtain the exact solution to the first step :

$$
u^{1}(x, y, t)=\lim _{m \rightarrow+\infty} \varphi_{m}(x, y, t)=e^{x y}(\cos t+\sin t)
$$

Second step

For $k=2$, we have:

$$
\begin{gathered}
N\left(u^{1}(x, y, t)\right)=(\cos t+\sin t)^{2} \frac{\partial^{2}}{\partial x \partial y}\left[\left(\frac{\partial^{2}\left(e^{x y}\right)}{\partial x^{2}}\right)\left(\frac{\partial^{2}\left(e^{x y}\right)}{\partial y^{2}}\right)\right]-(\cos t+\sin t)^{2} \frac{\partial^{2}}{\partial x \partial y}\left[(x y)\left(\frac{\partial\left(e^{x y}\right)}{\partial x}\right)\left(\frac{\partial\left(e^{x y}\right)}{\partial y}\right)\right] \\
=2(\cos t+\sin t)^{2} x y e^{2 x y}\left(2 x^{2} y^{2}+5 x y+2\right)-2(\cos t+\sin t)^{2} x y e^{2 x y}\left(2 x^{2} y^{2}+5 x y+2\right)=0 \\
\Rightarrow \tilde{N}\left(u^{1}(x, y, z)\right)=\int_{0}^{t}\left(\int_{0}^{s} N\left(u^{1}(x, y, z)\right) d z\right) d s=0
\end{gathered}
$$

From(24), we obtain :

$$
\begin{cases}u_{0}^{2} & =e^{x y}+t e^{x y} \\ u_{1}^{2} & =e^{x y}\left(-\frac{t^{2}}{2 !}-\frac{t^{3}}{3 !}\right) \\ u_{2}^{2} & =e^{x y}\left(\frac{t^{4}}{4 !}+\frac{t^{5}}{5 !}\right) \\ u_{3}^{2} & =e^{x y}\left(-\frac{t^{6}}{6 !}-\frac{t^{7}}{7 !}\right) \\ \cdot & \cdot \\ \cdot & =e^{x y}\left[(-1)^{n} \frac{t^{2 n}}{(2 n) !}+(-1)^{n} \frac{t^{2 n+1}}{(2 n+1) !}\right]\end{cases}
$$

then

$$
\varphi_{m}(x, y, t)=\sum_{n=0}^{m-1} u_{n}^{2}(x, y, t)=e^{x y}\left(\sum_{n=0}^{m-1}\left[(-1)^{n} \frac{t^{2 n}}{(2 n) !}+(-1)^{n} \frac{t^{2 n+1}}{(2 n+1) !}\right]\right)
$$

And the solution to the second step is

$$
u^{2}(x, y, t)=\lim _{m \rightarrow+\infty} \varphi_{m}(x, y, t)=e^{x y}(\cos t+\sin t)
$$

While using the same procedure for $k \geqslant 3$, the solution to the $k$ step is

$$
u^{k}(x, y, t)=\lim _{m \rightarrow+\infty} \varphi_{m}(x, y, t)=e^{x y}(\cos t+\sin t)
$$

So the exact solution of example 2 is:

$$
u(x, y, t)=\lim _{k \rightarrow+\infty} u^{k}(x, y, t)=e^{x y}(\cos t+\sin t)
$$

\section{Example 3}

Consider the following nonlinear system of partial differential equation (wazwaz, A.M., (2002)): 


$$
\left\{\begin{aligned}
u_{t}(x, y, t)+v_{x}(x, y, t) w_{y}(x, y, t)-v_{y}(x, y, t) w_{x}(x, y, t) & =-u(x, y, t) \\
v_{t}(x, y, t)+w_{x}(x, y, t) u_{y}(x, y, t)+w_{y}(x, y, t) u_{x}(x, y, t) & =v(x, y, t) \\
w_{t}(x, y, t)+u_{x}(x, y, t) v_{y}(x, y, t)+u_{y}(x, y, t) v_{x}(x, y, t) & =z(x, y, t) \\
u(x, y, 0) & =e^{x+y} \\
v(x, y, 0) & =e^{x-y} \\
w(x, y, 0) & =e^{-x+y}
\end{aligned}\right.
$$

let's put

$$
\left\{\begin{array}{l}
N_{1}(v(x, y, t), w(x, y, t))=-v_{x}(x, y, t) w_{y}(x, y, t)+v_{y}(x, y, t) w_{x}(x, y, t) \\
N_{2}(u(x, y, t), w(x, y, t))=-w_{x}(x, y, t) u_{y}(x, y, t)-w_{y}(x, y, t) u_{x}(x, y, t) \\
N_{3}(u(x, y, t), v(x, y, t))=-u_{x}(x, y, t) v_{y}(x, y, t)-u_{y}(x, y, t) v_{x}(x, y, t)
\end{array}\right.
$$

From (36), we have

$$
\left\{\begin{array}{l}
u(x, y, t)=u(x, y, 0)-\int_{0}^{t} u(x, y, s) d s+\int_{0}^{t} N_{1}(v(x, y, s), w(x, y, s)) d s \\
v(x, y, t)=v(x, y, 0)-\int_{0}^{t} v(x, y, s) d s+\int_{0}^{t} N_{2}(u(x, y, s), w(x, y, s)) d s \\
w(x, y, t)=w(x, y, 0)-\int_{0}^{t} w(x, y, s) d s+\int_{0}^{t} N_{3}(u(x, y, s), v(x, y, s)) d s
\end{array}\right.
$$

For every $k \geqslant 1$ we get $u_{n}^{k}(x, y, t)$ for $n \geqslant 0$, through the following SBA algorithm

$$
\begin{aligned}
& \left\{\begin{array}{l}
u_{0}^{k}=e^{x-y}+\tilde{N}_{1}\left(v^{k-1}, w^{k-1}\right) \\
u_{n}^{k}=-\int_{0}^{t} u_{n-1}^{k}(x, y, s) d s
\end{array}\right. \\
& \left\{\begin{array}{l}
v_{0}^{k}=e^{x-y}+\tilde{N}_{2}\left(u^{k-1}, w^{k-1}\right) \\
v_{n}^{k}=-\int_{0}^{t} v_{n-1}^{k}(x, y, s) d s
\end{array}\right. \\
& \left\{\begin{array}{l}
w_{0}^{k}=e^{x-y}+\tilde{N}_{3}\left(u^{k-1}, v^{k-1}\right) \\
w_{n}^{k}=-\int_{0}^{t} u_{n-1}^{k}(x, y, s) d s
\end{array}\right.
\end{aligned}
$$

With

$$
\left\{\begin{array}{l}
\tilde{N}_{1}\left(v^{k-1}, w^{k-1}\right)=\int_{0}^{t} N_{1}\left(v^{k-1}(x, y, s), w^{k-1}(x, y, s)\right) d s \\
\tilde{N}_{2}\left(u^{k-1}, w^{k-1}\right)=\int_{0}^{t} N_{2}\left(u^{k-1}(x, y, s), w^{k-1}(x, y, s)\right) d s \\
\tilde{N}_{3}\left(u^{k-1}, v^{k-1}\right)=\int_{0}^{t} N_{3}\left(u^{k-1}(x, y, s), v^{k-1}(x, y, s)\right) d s
\end{array}\right.
$$


For $k=1$,

Choosing $u^{0}=0, v^{0}=0, w^{0}=0$, we have $\tilde{N}_{1}\left(v^{0}, w^{0}\right)=\tilde{N}_{2}\left(u^{0}, w^{0}\right)=\tilde{N}_{3}\left(u^{0}, v^{0}\right)=0$,

From $(39,40,41)$,we obtain

$$
\left\{\begin{aligned}
u_{0}^{1} & =e^{x+y} \\
u_{1}^{1} & =-t e^{x+y} \\
u_{2}^{1} & =\frac{t^{2}}{2 !} e^{x+y} \\
u_{3}^{1} & =-\frac{t^{3}}{3 !} e^{x+y} \\
\cdot & \\
\cdot & \\
\cdot & \\
u_{n}^{1} & =\frac{(-1)^{n} t^{n}}{n !} e^{x+y}
\end{aligned}\right.
$$

$$
\left\{\begin{aligned}
v_{0}^{1} & =e^{x-y} \\
v_{1}^{1} & =t e^{x-y} \\
v_{2}^{1} & =\frac{t^{2}}{2} e^{x-y} \\
v_{3}^{1} & =\frac{t^{3}}{3 !} \frac{t^{2}}{2} e^{x-y} \\
\cdot & \\
\cdot & \\
\cdot & =\frac{t^{n}}{n !} e^{x-y}
\end{aligned}\right.
$$

$$
\left\{\begin{aligned}
w_{0}^{1} & =e^{-x+y} \\
w_{1}^{1} & =t e^{-x+y} \\
w_{2}^{1} & =\frac{t^{2}}{2} e^{-x+y} \\
w_{3}^{1} & =\frac{t^{3}}{3 !} \frac{t^{2}}{2} e^{-x+y} \\
\cdot & \\
\cdot & \\
\cdot & \\
w_{n}^{1} & =\frac{t^{n}}{n !} e^{-x+y}
\end{aligned}\right.
$$

Thus

$$
\left\{\begin{array}{c}
\varphi_{m}^{1}=\sum_{n=0}^{m-1} u_{n}^{1}(x, y, t)=\left(\sum_{n=0}^{m-1} \frac{(-1)^{n} t^{n}}{n !}\right) e^{x+y} \\
\phi_{m}^{1}=\sum_{n=0}^{m-1} u_{n}^{1}(x, y, t)=\left(\sum_{n=0}^{m-1} \frac{t^{n}}{n !}\right) e^{x-y} \\
\psi_{m}^{1}=\sum_{n=0}^{m-1} u_{n}^{1}(x, y, t)=\left(\sum_{n=0}^{m-1} \frac{t^{n}}{n !}\right) e^{-x+y}
\end{array}\right.
$$


either

$$
\left\{\begin{array}{c}
u^{1}(x, t)=\lim _{k \rightarrow+\infty} \varphi_{m}^{1}=e^{x+y-t} \\
v^{1}(x, t)=\lim _{k \rightarrow+\infty} \phi_{m}^{1}=e^{x-y+t} \\
w^{1}(x, t)=\lim _{k \rightarrow+\infty} \psi_{m}^{1}=e^{-x+y+t}
\end{array}\right.
$$

For $k=2$, We have :

$$
\left\{\begin{array}{l}
\tilde{N}_{1}\left(v^{1}, w^{1}\right)=\int_{0}^{t} N_{1}\left(v^{1}(x, y, s), w^{1}(x, y, s)\right) d s=\int_{0}^{t}\left(-\frac{\partial v^{1}}{\partial x} \times \frac{\partial w^{1}}{\partial y}+\frac{\partial v^{1}}{\partial y} \times \frac{\partial w^{1}}{\partial x}\right) d s=\int_{0}^{t}\left(-e^{2 s}+e^{2 s}\right) d s=0 \\
\tilde{N}_{2}\left(u^{1}, w^{1}\right)=\int_{0}^{t} N_{2}\left(u^{1}(x, y, s), w^{1}(x, y, s)\right) d s=\int_{0}^{t}\left(-\frac{\partial w^{1}}{\partial x} \times \frac{\partial u^{1}}{\partial y}-\frac{\partial w^{1}}{\partial y} \times \frac{\partial u^{1}}{\partial x}\right) d s=\int_{0}^{t}\left(e^{2 y}-e^{2 y}\right) d s=0 \\
\tilde{N}_{3}\left(u^{1}, v^{1}\right)=\int_{0}^{t} N_{3}\left(u^{1}(x, y, s), v^{1}(x, y, s)\right) d s=\int_{0}^{t}\left(-\frac{\partial u^{1}}{\partial x} \times \frac{\partial v^{1}}{\partial y}-\frac{\partial u^{1}}{\partial y} \times \frac{\partial v^{1}}{\partial x}\right) d s=\int_{0}^{t}\left(e^{2 x}-e^{2 x}\right) d s=0
\end{array}\right.
$$

From $(39,40,41)$,we obtain

$$
\begin{aligned}
& \left\{\begin{aligned}
u_{0}^{2} & =e^{x+y} \\
u_{1}^{2} & =-t e^{x+y} \\
u_{2}^{2} & =\frac{t^{2}}{2 !} e^{x+y} \\
u_{3}^{2} & =-\frac{t^{3}}{3 !} e^{x+y} \\
\cdot & \\
\cdot & \\
\cdot & \\
u_{n}^{2} & =\frac{(-1)^{n} t^{n}}{n !} e^{x+y}
\end{aligned}\right. \\
& \left\{\begin{aligned}
v_{0}^{2} & =e^{x-y} \\
v_{1}^{2} & =t e^{x-y} \\
v_{2}^{2} & =\frac{t^{2}}{2} e^{x-y} \\
v_{3}^{2} & =\frac{t^{3}}{3 !} \frac{t^{2}}{2} e^{x-y} \\
\cdot & \\
\cdot & \\
\cdot & =\frac{t^{n}}{n !} e^{x-y}
\end{aligned}\right. \\
& \left\{\begin{aligned}
w_{0}^{2} & =e^{-x+y} \\
w_{1}^{2} & =t e^{-x+y} \\
w_{2}^{2} & =\frac{t^{2}}{2} e^{-x+y} \\
w_{3}^{2} & =\frac{t^{3}}{3 !} \frac{t^{2}}{2} e^{-x+y} \\
\cdot & \\
\cdot & \\
\cdot & \\
w_{n}^{2} & =\frac{t^{n}}{n !} e^{-x+y}
\end{aligned}\right.
\end{aligned}
$$


then

$$
\left\{\begin{array}{c}
\varphi_{m}^{2}=\sum_{n=0}^{m-1} u_{n}^{2}(x, y, t)=\left(\sum_{n=0}^{m-1} \frac{(-1)^{n} t^{n}}{n !}\right) e^{x+y} \\
\phi_{m}^{2}=\sum_{n=0}^{m-1} u_{n}^{2}(x, y, t)=\left(\sum_{n=0}^{m-1} \frac{t^{n}}{n !}\right) e^{x-y} \\
\psi_{m}^{2}=\sum_{n=0}^{m-1} u_{n}^{2}(x, y, t)=\left(\sum_{n=0}^{m-1} \frac{t^{n}}{n !}\right) e^{-x+y}
\end{array}\right.
$$

either

$$
\left\{\begin{array}{c}
u^{2}(x, t)=\lim _{k \rightarrow+\infty} \varphi_{m}^{2}=e^{x+y-t} \\
v^{2}(x, t)=\lim _{k \rightarrow+\infty} \phi_{m}^{2}=e^{x-y+t} \\
w^{2}(x, t)=\lim _{k \rightarrow+\infty} \psi_{m}^{2}=e^{-x+y+t}
\end{array}\right.
$$

While using the same procedure for $k \geqslant 3$, the solution to the $k$ step is

$$
\left\{\begin{array}{r}
u^{k}(x, t)=\lim _{k \rightarrow+\infty} \varphi_{m}=e^{x+y-t} \\
v^{k}(x, t)=\lim _{k \rightarrow+\infty} \phi_{m}=e^{x-y+t} \\
w^{k}(x, t)=\lim _{k \rightarrow+\infty} \psi_{m}=e^{-x+y+t}
\end{array}\right.
$$

from which,we obtain

$$
\left\{\begin{array}{r}
u(x, t)=\lim _{k \rightarrow+\infty} u^{k}(x, t)=e^{x+y-t} \\
v(x, t)=\lim _{k \rightarrow+\infty} v^{k}(x, t)=e^{x-y+t} \\
w(x, t)=\lim _{k \rightarrow+\infty} w^{k}(x, t)=e^{-x+y+t}
\end{array}\right.
$$

\section{Conclusion}

Through these example,we showed again the usefulness of the SBA method,in the research of an approximate solution of an equation and this method give us the exact solution.

\section{References}

Abbo, B. (2007). Nouvel algorithme numérique de ésolution des équations différentielles ordinaires (EDO) et des équations aux dérivées partielles non linéaires These de Doctorat unique. Université de Ouagadougou.

Bonazebi, Y. J., Pare, Y., Bissanga, G., Bassono, F., \& Some, B. (2014). Application of the Adomian Decomposition Method (ADM) and the SOME BLAISE ABBO (SBA) method to solving the diffusion-reaction equations. Advances in Theoretical and Applied Mathematics, 9(2), 97-104.

Pare, Y. (2010). Rsolution de quelques quations fonctionnelles par la mthode SBA (Som Blaise-Abbo). Thse de Doctorat unique, Universit de Ouagadougou.

Pare, Y., Yaro, R., Elysée, G., \& Blaise, S. (2012) Solving a system of nonlinear equations second kind of Volterra by the SBA method, Far.East.J.Appl. Maths*., 71(1), 43-54.

Ghoreishi, M., Ismail, A. I. B., \& Ali, N. H. M. (2010). Adomian decomposition method for nonlinear wave-like equation with variable coefficients. Applied Mathematical Sciences, 4, 2431-2444.

Gupta, V. G., \& Sumit, G. (2013). Homotopy perturbation transform method for solving nonlinear wave-like equation with variable coefficients. Journal of International and Computing Science, 8(3), 163-172.

Wazwaz, A. M. (2002). Partial differential equations methods and applications. Netherland Balkema Publisher. 


\section{Copyrights}

Copyright for this article is retained by the author(s), with first publication rights granted to the journal.

This is an open-access article distributed under the terms and conditions of the Creative Commons Attribution license (http://creativecommons.org/licenses/by/4.0/). 